\title{
Clock Compensation Strategy in Train Ethernet
}

\author{
Xiaoyong Zhang, Jun Peng, Shuo Li \\ School of Information Science and Engineering \\ Central South University \\ Changsha, China \\ zhangxy@csu.edu.cn
}

\begin{abstract}
How to degrade the delay time of gateway queuing with heavy network load in train communication networks is the key to guarantee the stability of the train. This article models the queue scheduling problem modeling into reinforcement learning process, puts forward an adaptive weights learning polling scheduling algorithm with the purpose of dynamic scheduling for vehicle gateway node queue. With the compare of algorithm in this paper and weighted roundrobin scheduling algorithm in adequate and inadequate bandwidth resources situations, we have approved the superiority of the algorithm in this paper.
\end{abstract}

Keywords-component; clock synchronization; Kalman filtering; clock compensation

\section{INTRODUCTION}

In order to ensure the instantaneity of train Ethernet and satisfy the high precision clock synchronization, IEEE1588 agreement is generally used. The entire network inside the clock is divided into two categories: OC (Ordinary Clock) and $\mathrm{BC}$ (Boundary Clock) $[1,2]$. Using IEEE1588 clock synchronization protocol for time synchronization process includes two stages: Offset measurement and delay measurement. But due to the complicated environment of network, clock node will show a little deviation. The error of clock synchronization can be classified into three categories: time deviation, frequency deviation and frequency drift. For the convenience of analysis, this paper introduces a state variable model to analyze and describe the node clock, State variable model was adopted in many previous study, such as state variable model for the clock mode[3][4]. Clock compensation is aimed at the time and frequency of these two variables. In paper [5], the author analyzed the state variable's model by two state variables. And this model is also used in the analysis of the mathematical model for embedded system clock management module, which is also adopted by this paper.

To solve this problem, time bias is needed, i.e. jet lag compensation mechanism and indirect Kalman filter frequency clock compensation mechanism. On that basis, this paper proposed an indirect compensation strategy based on IEEE 1588 clock synchronization protocol. From the mathematical analysis and the simulation results we know that, Clock's difference accuracy is improved because of noise reduction processing via Kalman filter for clock deviation, which improves the accuracy of the compensation results and makes the trend of clock deviation smoother. In this way, we improve the clock deviation and the stability of the system at the same time.

\section{SYSTEM MODEL}

\section{A. Time synchronization model for train communication networks}

The ordinary clock in IEEE 1588 agreement has only one IEEE 1588 communication port, but boundary clock has more than one. So BC clock's port connecting primary clock can be thought as the slave clock, thus we update and adjust the local clock after receiving master clock's synchronous message, and synchronize all the OC connected clock after the adjustment. In this way the connection topology becomes simple point-to-point connections, thus we can remove the delay jitter effectively. Theoretically, any clock can act as master and slave clock, but a communication subnet can only have one master clock. So master clock usually use the server with high performance and reliability for implementation, this paper assume that master clock node is stable, and can provide the accurate time, so we can study the problem of node time deviation compensation.

According to the IEEE 1588 agreement, to synchronize train communication network time, four kinds of basic synchronous data datagram is needed: SYNC, Follow_UP, Delay_Rep and Delay_Resp.

\section{B. Clock synchronization process}

Offset measurement is used to correct the time deviation, and this is shown in Fig. 1.

Every two seconds the master clock node broadcast the radio synchronous message SYNC to every needed network. The message includes the estimating time when message leaves master clock, and meanwhile the master clock transfers the accurate measurement time Tm1. Then the slave node receives SYNC message and records accurate time Ts1. And master clock node send follow Follow_UP message, which associates with SYNC message and contains more accurate time estimation of Tm1. From these we can calculate in (1), and the means the delay in transmission, its default value is 0 .

$$
\text { Offset }=T s 1-\text { Tm1 }- \text { Delay }
$$

Besides, the slave node adjusts time according to the calculated offset time:

$$
\text { AdjustTime }=T s-\text { Offset }
$$

After the second time synchronization process, masterslave clock has achieved synchronization without considering the line propagation delay. 


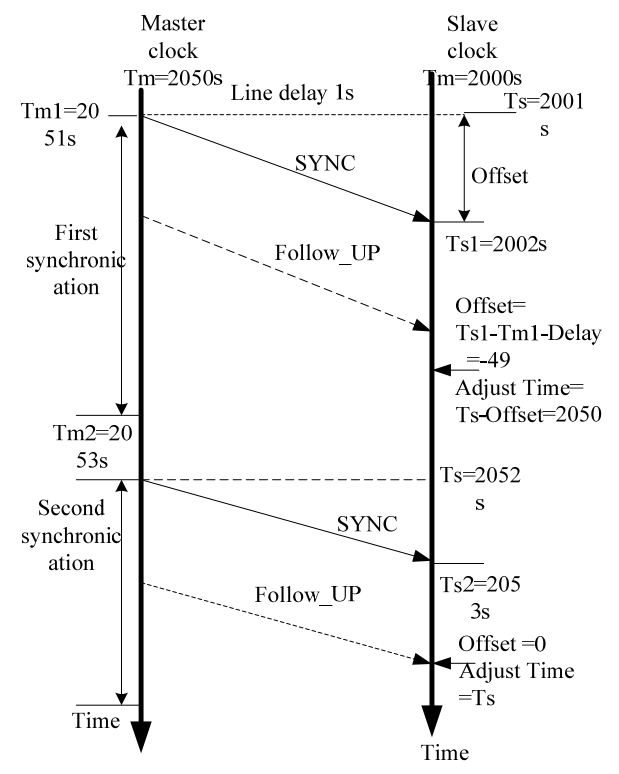

Figure 1. Timing sequence diagram for offset synchronization measurement in train communication networks

Delay measurement is used for measuring the propagation between master-slave clocks. In order to improve the precision of the synchronization, we need the second stage of delay measurement, measuring the send and receive delay between master-slave clocks. The measuring step is as follows.

Firstly slave node sends delay measurement request message Delay_Rep to the master node, and records the exact time of the sending time Ts3; Then the master node records accurate receiving time $\mathrm{Tm} 3$ after received message Delay_Rep, and sends delay measurement request data message Delay Resp to slave node, in which contains message Tm3; Lastly after receiving Delay_Resp, we calculate the line Delay according to (3).

$$
\text { Delay }=T m 3-T s 3+\text { Offset }
$$

In conclusion, The protocol of accurate clock synchronization draws the time deviation Offset and interconnect Delay value through exchanging timestamp information data message, but the premise is that the link propagation delay is symmetric, which means transmission delay between master clock to and slave clock $d_{m s}$ is the same.

$$
\begin{gathered}
d_{m s}=T_{s 1}-T_{m 1} \\
d_{s m}=T_{m 3}-T_{s 3} \\
\text { Delay }=\frac{d_{m s}+d_{s m}}{2}=\frac{\left(T_{s 1}-T_{m 1}\right)+\left(T_{m 3}-T_{s 3}\right)}{2} \\
\text { Offset }=d_{m s}-\text { Delay }=\frac{\left(T_{s 1}-T_{m 1}\right)-\left(T_{m 3}-T_{s 3}\right)}{2}
\end{gathered}
$$

According to the calculation, after the subsequent two synchronous processes, the master and slave clock can achieve synchronization.

\section{COMPENSATION MECHANISM RESEARCH}

\section{A. Train clock synchronous model and error classification}

Clock synchronization error can be classified into three categories: time deviation, frequency deviation and frequency drift. Then we can define the following err expressions, which $C(t)$ means the time recorded at $T_{n}$, and $T_{n}=t$.

Time deviation: Time deviation between clock time and standard time.

$$
\theta(t)=C(t)-t
$$

Frequency deviation: It's usually used to measure frequency deviation degree.

$$
\gamma_{i}=\frac{f_{i}-f_{T}}{f_{T}}=\frac{f_{i}}{f_{T}}-1=C^{\prime}(t)-t^{\prime}=\theta^{\prime}
$$

Frequency drift: long-term frequency change caused by the change of the environment. So in this paper, we don't take it into consideration.

For convenience, this paper adopts two variables state model for analysis. Clock management module's output voltage is given by $(10)$, in which $f_{T}$ is the clock counting frequency setting by programming, $\Phi_{0}$ is the initial phase, $\Phi(\mathrm{t})$ is affecting the time precision of stochastic process.

$$
V(t)=V_{0} \cos 2 \pi\left(f_{T} t+\frac{\Phi}{2 \pi}\right)
$$

Due to the phase noise has no effect on IEEE 1588 agreement based synchronization method, so we can get time deviation discrete recursive expression in (11).

$$
\theta(n+1)=\theta(n)+\gamma(n) \Delta T
$$

where $\Delta T=T_{n}-T_{n-1}$.

From (8), we can get the equation of continuous time synchronization of recursive state as (12) and (13). Among them $\omega_{\theta}(n)$ and $\omega_{\gamma}(n)$ are two independent stochastic process noise with Gaussian distribution, and the variance is $\delta_{\theta}^{2} \Delta T$ and $\delta_{\gamma}^{2} \Delta T$.

$$
\begin{gathered}
\theta(n+1)=\theta(n)+\gamma(n) \Delta T+\omega_{\theta}(n) \\
\gamma(n+1)=\gamma(n)+\omega_{\gamma}(n)
\end{gathered}
$$

The two types studied above in this paper constitute the compensation mechanism for clock synchronization, this model has three constant parameters : Time difference $\Delta T$ when master clock node sending synchronous message persistently; Two noise parameters $\delta_{\theta}^{2} \Delta T$ and $\delta_{\gamma}^{2} \Delta T$, we take various factors as noise which influencing clock crystal oscillator and time accuracy, and its numerical value is decided by clock model component noise properties.

\section{B. Kalman filter frequency indirect clock compensation mechanism}

Direct time differential compensation mechanism compensate the clock time after calculate the clock deviation. But in the distributed network control system, the direct compensation will make from node clock mutate and larger fluctuation, appear "time fast forward" and "time upstream" unstable phenomenon. To solve this problem, this paper puts 
forward the indirect Kalman filter frequency time compensation mechanism.

In order to achieve master and slave clock synchronization, in the time $\mathrm{n}$, we put time-difference correction $u_{\theta}(n)$ and $u_{\gamma}(n)$ deviation rate correction into clock state model established by formula (12) and (13), and we get (14) and (15).

$$
\begin{gathered}
\theta(n+1)=\theta(n)-u_{\theta}(n)+\left[\gamma(n)-u_{\gamma}(n)\right] \Delta T+\omega_{\theta}(n) \\
\gamma(n+1)=\gamma(n)-u_{\gamma}(n)+v(n)
\end{gathered}
$$

Due to interference, we can't get accurate time and frequency partial information, so this paper uses Kalman filter method for frequency compensatory pretreatment. Then we can write two combinations above in matrix form as (16).

$$
x(n)=A x(n-1)+B u(n-1)+\omega(n-1)
$$

where $u(n)$ is the input vector, $x(n)$ is the state vector, $\mathrm{A}$ and $\mathrm{B}$ is the Sync synchronous message.

$$
\begin{gathered}
u(n)=\left[u_{\theta}(n), u_{\gamma}(n)\right]^{T} \\
x(n)=[\theta(n) \gamma(n)]^{T} \\
A=\left[\begin{array}{cc}
1 & \Delta T \\
0 & 1
\end{array}\right] \\
B=\left[\begin{array}{cc}
-1 & -\Delta T \\
0 & -1
\end{array}\right] .
\end{gathered}
$$

And $\theta_{M}(n)$ is the time measuring deviation at time $\mathrm{n}$, at the same time $\gamma_{M}(n)$ is the frequency measuring deviation rate:

$$
\gamma_{M}(n)=\frac{\theta_{M}(n)-\theta_{M}(n-1)}{\Delta T}
$$

Thus we can define the measurement equation of Kalman filter :

$$
z(n)=H x(n)+v(n)
$$

In which $z(n)=\left[\theta_{M}(n) \gamma_{M}(n)\right]^{T} ; H$ is the unit matrix, shows the gain of measurement vector from state vector ; $v(n)$ is the noice of measurement process, and it's a Gaussian distributed random variables whose mean value is 0,besides, it's composited by two zero mean value random variables $v_{\theta M}(n)$ and $v_{\gamma M}(n)$, and respectively the variance are $\sigma_{\theta M}^{2}$ and $\sigma_{\gamma M}^{2}$.

After that we get five Kalman filtering equations ${ }^{[7]}$, Equation (23) and (24) are Kalman filter predicted equations. Among them, $\hat{x}(n \mid n-1)$ is the prediction of transcendental state; $P(n \mid n-1)$ is the prediction of transcendental state; is a posteriori error covariance estimation; Matrix $Q$ presents the process noise covariance matrix; Because random process $\gamma(n)$ and $\theta(n)$ are not related, so $Q$ is a second order diagonal matrix, and it's Nonzero diagonal element are $\delta_{\theta}^{2} \Delta T$ and $\delta_{\gamma}^{2} \Delta T$.

$$
\begin{aligned}
& \hat{x}(n \mid n-1)=A \hat{x}(n-1)+B u(n-1) \\
& P(n \mid n-1)=A P(n-1) A^{T}+Q
\end{aligned}
$$

Besides, Kalman filter gain is show in (25), and Kalman filter correction process equation can be defined as (26) and (27).

$$
\begin{gathered}
K(n)=P(n \mid n-1)[P(n \mid n-1)+R]^{-1} \\
\hat{x}(n)=\hat{x}(n \mid n-1)+K(n)[z(n)-\hat{x}(n \mid n-1)] \\
P(n)=[I-K(n)] P(n \mid n-1)
\end{gathered}
$$

And $R$ is a second order measurement noise covariance matrix.

$$
R=\left[\begin{array}{cc}
\delta_{\theta M}^{2} & \frac{\delta_{\theta M}^{2}}{\Delta T} \\
\frac{\delta_{\theta M}^{2}}{\Delta T} & 2\left(\frac{\delta_{\theta M}}{\Delta T}\right)^{2}
\end{array}\right]
$$

Which Clock compensation input can be set as : $u(n)=\widehat{x}(n)$.

After getting the estimation of deviation ratio we can adjust the timing frequency and node frequency of slave clock management unit. Assuming that at time $n-1$ the master and slave clock get synchronization, and they produce time deviation at time $n$. At the same time we set $\hat{f}_{s}(n)$ as frequency estimation.

$$
\hat{f}_{s}=[\gamma(n)+1] f_{T}
$$

Via the clock synchronization protocol for synchronous message switching, we set the slave clock time management unit frequency as :

$$
f_{s}=\frac{f_{T}[\Delta T-\theta(n)]}{\Delta T}
$$

And the slave node frequency compensation value is set as :

$$
\begin{aligned}
& \Delta f_{s}=\left\{\begin{array}{lc}
\frac{f_{T}[\Delta T-\theta(n)]}{\Delta T}-\widehat{f}_{s} & (\theta(n)<0) \\
\hat{f}_{s}-\frac{f_{T}[\Delta T-\theta(n)]}{\Delta T} & (\theta(n)>0)
\end{array}\right. \\
& \Delta f_{s}= \begin{cases}f_{T}\left[\frac{\theta(n)}{\Delta T}-\gamma(n)\right] & (\theta(n)<0) \\
f_{T}\left[\frac{\theta(n)}{\Delta T}+\gamma(n)\right] & (\theta(n)>0)\end{cases}
\end{aligned}
$$

Via calculating time stamp from time synchronization exchanging and synchronous message, then we can calculate the measured value $\theta_{M}(n)$ of deviation ratio. Taking Kalman filter's output evaluation value as an adjustment reference, we adjust the frequency of slave clock according to equation (30) and achieve the goal of reducing time deviation. Due to the existence of environment temperature, electromagnetic interference, frequency adjustment cannot reach the designated position in one step, gradually in a reciprocating cycle; the whole frequency adjustment tends towards the master clock's frequency, so as to reach the purpose of indirect time compensation.

\section{SimUlation AND ANALysis}

In this section, we study the master-slave clock node clock synchronization method in Matlab simulation analysis. So we set the initial conditions as: The initial time error is 0 , 
and $\delta_{\theta}^{2}=10^{-5} s^{2}, \delta_{\theta}^{2}=10^{-5} s^{2}$. Slave clock process a synchronous in every $100 \mathrm{~ms}$ and a time measurement in every $1 \mathrm{~ms}$. Supposing that the clock drift is random, the clock produces clock drift for $\pm 30 \mu s$ in every $100 \mathrm{~ms}$. Besides, in the first simulation of $200 \mathrm{~ms}$, we don't use any clock compensation method, then we process a compensation adjustment in every $100 \mathrm{~ms}$. We estimate the value of clock synchronization performance from the attitude of master-slave clock deviation and respectively compare the simultaneous effects.

The contrast of before and after indirect differential frequency compensation are shown in Fig.2. In the first $200 \mathrm{~ms}$, the value of master-slave clock deviation is greater because of no clock compensation. Then in every $100 \mathrm{~ms}$ the clock processes direct compensation and frequency compensation respectively, and due to the noise interference, the clock deviation value is not stable and with small jitter.

In Fig.3., direct clock compensation cause time mutation due to direct adjustment in every compensation, and meanwhile with no adjust to clock frequency, masterslave clock deviation increases in each compensation gradually. On the contrary, frequency compensation made adjust clock counting frequency for compensation. The master-slave clock time deviation reduces gradually.

Comparing the two kinds of mode, we can see that the accuracy of frequency compensation clock synchronization is improved through its slower. In $400 \mathrm{~ms}$, after four times compensation, the range of clock error has been less than $0.2 \mathrm{~ms}$, while with the direct compensation the clock error range are still float up and down in $1.8 \mathrm{~ms}$.

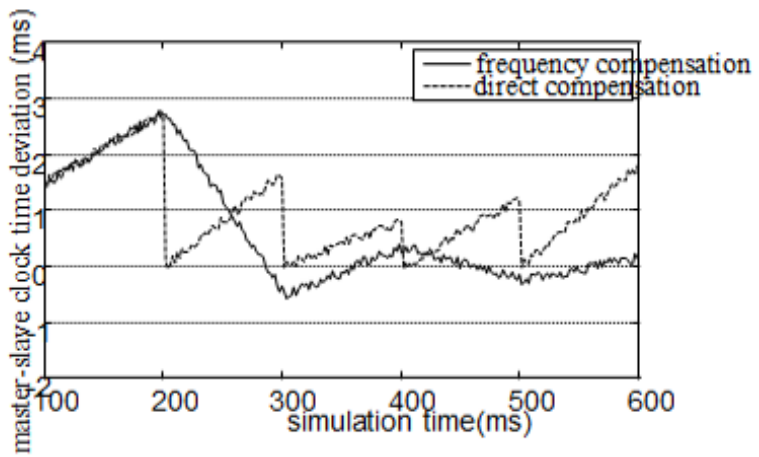

Figure 2. Clock bias of direct compensation and frequency compensation

At the same time, because frequency compensation changes continuously, we can guarantee the stability of train communication network.

This simulation compares the clock bias with Kalman filter and without Kalman filter. As shown in Fig. 3 below, from $200 \mathrm{~ms}$, the accuracy of clock deviation value is improved after introducing Kalman filter for noise reduction processing, making compensation results more accurate and smooth.

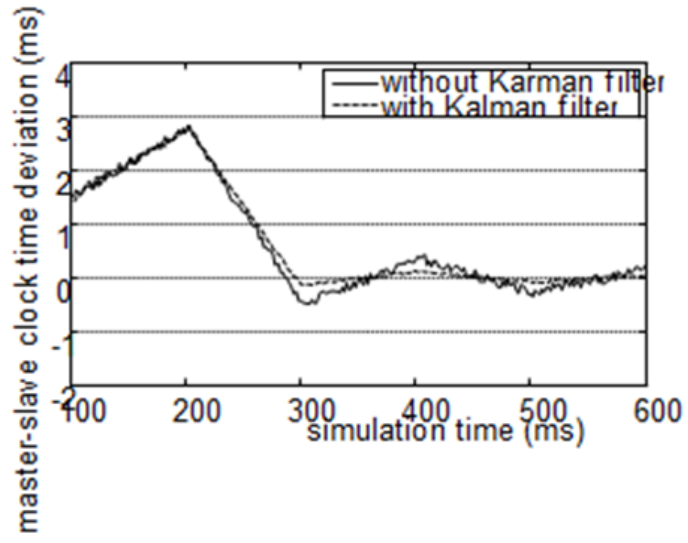

Figure 3. With and without Kalman filter frequency compensation clock bias

\section{CONCLUSION}

In this paper, we take a thorough research on the precise time synchronization technology in train Ethernet, analyze the reasons for the instability of direct clock compensation and put forward a kind of clock frequency indirect compensation method using Kalman filtering. This method reduces noise through the filtering processing for the synchronous acquisition information, which improves the accuracy of the data. Then we compensate the clock counting frequency. In this way, we improve the indirect clock deviation and improve the accuracy of the clock synchronization at the same time, which ensures the stability of the system.

\section{REFERENCES}

[1] IEEE Std 1588-2002. IEEE standard for a precision clock synchronization protocol for network measurement and control systems. New York: Institute of Electrical and Electronic Engineers, 2002.

[2] IEEE Std 1588-2008, IEEE standard for a precision clock synchronization protocol for network measurement and control systems. New York: Institute of Electrical and Electronic Engineers, 2008.

[3] G. Giorgi, "Performance analysis of kalman-filter-based clock synchronization in IEEE 1588 networks". IEEE Transactions on Instrumentation and Measurement, 2011, 60(8): 2902-2909.

[4] L. Scheiterer, "Synchronization performance of the precision time protocol in industrial automation networks". IEEE Transactions on Instrumentation and Measurement, 2009, 58(6): 1849-1857.

[5] E. Fuchs. "The future of silicon photonics: not so fast insights from $100 \mathrm{G}$ ethernet LAN transceivers". IEEE Journal of Lightwave Technology, 2011, 29(15): 2319-2326.

[6] W. Ye, "IEEE1588 clock servo algorithm. 9th International Conference on Electronic Measurement \& Instruments", 2009: 158165.Welch G., Bishop G.. An introduction to the kalman filter. Department of Computer Science, University of North Carolina, July 2006. 